\title{
On generalized Lagrange-Hermite-Bernoulli and related polynomials
}

\author{
Waseem A. Khan and M. A. Pathan
}

\begin{abstract}
We introduce a new class of generalized polynomials, ascribed to the family of Hermite, Lagrange, Bernoulli, Miller-Lee, and Laguerre polynomials and of their associated forms. These polynomials can be expressed in the form of generating functions, which allow a high degree of flexibility for the formulation of the relevant theory. We develop a point of view based on generating relations, exploited in the past, to study some aspects of the theory of special functions. We propose a fairly general analysis allowing a transparent link between different forms of special polynomials.
\end{abstract}

\section{Introduction}

The Lagrange polynomials in several variables, which are known as the Chan-Chyan-Srivastava polynomials [2], are defined by means of the generating function

$$
\prod_{j=1}^{r}\left(1-x_{j} t\right)^{-\alpha_{j}}=\sum_{n=0}^{\infty} g_{n}^{\left(\alpha_{1} \ldots \alpha_{r}\right)}\left(x_{1}, \ldots, x_{r}\right) t^{n}
$$

where $\alpha_{j} \in \mathbb{C}(j=1, \ldots, r) ;|t|<\min \left\{\left|x_{1}\right|^{-1}, \ldots,\left|x_{r}\right|^{-1}\right\}$. These polynomials are represented by

$$
\begin{gathered}
g_{n}^{\left(\alpha_{1}, \alpha_{2} \ldots \alpha_{r}\right)}\left(x_{1}, x_{2}, \ldots, x_{r}\right)=\sum_{k_{r-1}=0}^{n} \ldots \sum_{k_{2}=0}^{k_{3}} \sum_{k_{1}=0}^{k_{2}}\left(\alpha_{1}\right)_{k_{1}}\left(\alpha_{2}\right)_{k_{2}-k_{1}} \ldots \\
\left(\alpha_{r-1}\right)_{k_{r-1}-k_{r-2}}\left(\alpha_{r}\right)_{n-k_{r-1}} \frac{x_{1}^{k_{1}}}{k_{1} !} \frac{x_{2}^{k_{2}-k_{1}}}{\left(k_{2}-k_{1}\right) !} \ldots \frac{x_{r-1}^{k_{r}-k_{r-2}}}{\left(k_{r-1}-k_{r-2}\right) !} \frac{x_{r}^{n-k_{r}-1}}{\left(n-k_{r}-1\right) !}
\end{gathered}
$$

where $(\lambda)_{0}:=1$ and $(\lambda)_{n}=\lambda(\lambda+1) \ldots(\lambda+n-1) \quad(n \in \mathbb{N}:=\{1,2, \ldots\})$.

Received July 15, 2018.

2010 Mathematics Subject Classification. 11B68; 33C45.

Key words and phrases. Bernoulli polynomials; Laguerre polynomials; Hermite polynomials; Lagrange-Hermite polynomials; Lagrange-Hermite-Bernoulli polynomials.

https://doi.org/10.12697/ACUTM.2019.23.19 
Altin and Erkus [1] presented a multivariable extension of the so called Lagrange-Hermite polynomials generated by (see [1, p. 239])

$$
\prod_{j=1}^{r}\left(1-x_{j} t^{j}\right)^{-\alpha_{j}}=\sum_{n=0}^{\infty} h_{n}^{\left(\alpha_{1}, \ldots, \alpha_{r}\right)}\left(x_{1}, \ldots x_{r}\right) t^{n}
$$

where $\alpha_{j} \in \mathbb{C}(j=1, \ldots, r),|t|<\min \left\{\left|x_{1}\right|^{-1},\left|x_{2}\right|^{-\frac{1}{2}}, \ldots,\left|x_{r}\right|^{-\frac{1}{r}}\right\}$, and

$$
h_{n}^{\left(\alpha_{1}, \ldots, \alpha_{r}\right)}\left(x_{1}, \ldots, x_{r}\right)=\sum_{k_{1}+2 k_{2}+\cdots+r k_{r}=n}\left(\alpha_{1}\right)_{k_{1}} \ldots\left(\alpha_{r}\right)_{k_{r}} \frac{x_{1}^{k_{1}}}{k_{1} !} \ldots \frac{x_{r}^{k_{r}}}{k_{r} !} .
$$

The special case when $r=2$ in (1.3) is essentially a case which corresponds to the familiar (two-variable) Lagrange-Hermite polynomials considered by Dattoli et al. [4]:

$$
\left(1-x_{1} t\right)^{-\alpha_{1}}\left(1-x_{2} t^{2}\right)^{-\alpha_{2}}=\sum_{n=0}^{\infty} h_{n}^{\left(\alpha_{1}, \alpha_{2}\right)}\left(x_{1}, x_{2}\right) t^{n} .
$$

The multivariable (Erkus-Srivastava) polynomials $U_{n, l_{1}, \ldots, l_{r}}^{\left(\alpha_{1}, \ldots, \alpha_{r}\right)}\left(x_{1}, \ldots, x_{r}\right)$, defined by the generating function [5, p. 268]

$$
\prod_{j=1}^{r}\left(1-x_{j} t^{l_{j}}\right)^{-\alpha_{j}}=\sum_{n=0}^{\infty} U_{n, l_{1}, \ldots, l_{r}}^{\left(\alpha_{1} \ldots \alpha_{r}\right)}\left(x_{1}, \ldots, x_{r}\right) t^{n}
$$

where $\alpha_{j} \in \mathbb{C}(j=1, \ldots, r), l_{j} \in \mathbb{N}(j=1, \ldots, r), \quad|t|<\min \left\{\left|x_{1}\right|^{-1}\right.$ $\left., \ldots,\left|x_{r}\right|^{-1}\right\}$, are a unification (and generalization) of several known families of multivariable polynomials. It is evident that the Chan-ChyanSrivastava polynomials $g_{n}^{\left(\alpha_{1} \ldots \alpha_{r}\right)}\left(x_{1}, \ldots, x_{r}\right)$ and the Lagrange-Hermite polynomials $h_{n}^{\left(\alpha_{1} \ldots \alpha_{r}\right)}\left(x_{1}, \ldots, x_{r}\right)$ are special cases of the ErkusSrivastava polynomials $U_{n, l_{1} \ldots, l_{r}}^{\left(\alpha_{1}, \ldots, \alpha_{r}\right)}\left(x_{1}, \ldots, x_{r}\right)$ when $l_{j}=1(j=1, \ldots, r)$.

The generating function (1.5) yields the following explicit representation (see [5, p. 268]):

$$
U_{n, l_{1}, \ldots, l_{r}}^{\left(\alpha_{1}, \ldots, \alpha_{r}\right)}\left(x_{1}, \ldots, x_{r}\right)=\sum_{k_{1}, \ldots, k_{r} \in \mathbb{N}_{0},\left(l_{1} k_{1}+\cdots+l_{r} k_{r}=n\right)}\left(\alpha_{1}\right)_{k_{1}} \ldots\left(\alpha_{r}\right)_{k_{r}} \frac{x_{1}^{k_{1}}}{k_{1} !} \ldots \frac{x_{r}^{k_{r}}}{k_{r} !},
$$

which, in the special case $l_{j}=1(j=1, \ldots, r)$, corresponds to (1.2).

\section{Definitions and basic properties of}

\section{Lagrange-Hermite-Bernoulli and related polynomials}

In this section, we link the Lagrange polynomials in several variables with Hermite and Bernoulli polynomials. The resulting formulae allow a considerable unification of various special results which appear in the literature. 
Following Pathan [6[ and Pathan and Khan [7], it seems quite appropriate to define Lagrange-Hermite-Bernoulli polynomials ${ }_{B} H_{n}^{\left(\alpha_{1}, \ldots, \alpha_{r}\right)}\left(x \mid x_{1}, \ldots x_{r}\right)$ in several variables by means of the generating function

$$
\frac{t}{e^{t}-1} e^{x t} \prod_{j=1}^{r}\left(1-x_{j} t^{j}\right)^{-\alpha_{j}}=\sum_{n=0}^{\infty}{ }_{B} H_{n}^{\left(\alpha_{1}, \ldots, \alpha_{r}\right)}\left(x \mid x_{1}, \ldots x_{r}\right) t^{n},
$$

which, for ordinary case $r=2$, reduces to the Lagrange-Hermite-Bernoulli polynomials defined by

$$
\frac{t}{e^{t}-1} e^{x t}\left(1-x_{1} t\right)^{-\alpha_{1}}\left(1-x_{2} t^{2}\right)^{-\alpha_{2}}=\sum_{n=0}^{\infty}{ }_{B} H_{n}^{\left(\alpha_{1}, \alpha_{2}\right)}\left(x \mid x_{1}, x_{2}\right) t^{n} .
$$

In particular, when $x_{1}=x_{2}=0,(2.1)$ reduces to Bernoulli polynomials $B_{n}(x)$ defined by

$$
\frac{t}{e^{t}-1} e^{x t}=\sum_{n=0}^{\infty} B_{n}(x) \frac{t^{n}}{n !}
$$

The Lagrange-Hermite-Bernoulli polynomials of order $\alpha$ are defined by

$$
\left(\frac{t}{e^{t}-1}\right)^{\alpha} e^{x t}\left(1-x_{1} t\right)^{-\alpha_{1}}\left(1-x_{2} t^{2}\right)^{-\alpha_{2}}=\sum_{n=0}^{\infty}{ }_{B} H_{n}^{\left(\alpha \mid \alpha_{1}, \alpha_{2}\right)}\left(x \mid x_{1}, x_{2}\right) t^{n},
$$

so that

$$
{ }_{B} H_{n}^{\left(\alpha \mid \alpha_{1}, \alpha_{2}\right)}\left(x \mid x_{1}, x_{2}\right)=\sum_{m=0}^{n} h_{m}^{\left(\alpha_{1}, \alpha_{2}\right)}\left(x_{1}, x_{2}\right) \frac{B_{n-m}^{(\alpha)}(x)}{(n-m) !} .
$$

Setting $\alpha=x=0$ in (2.3), the result reduces to (1.4). For $\alpha=1$, the equality (2.3) reduces to $(2.1)$.

The Lagrange-Bernoulli polynomials of order $\alpha$ are defined by

$$
\left(\frac{t}{e^{t}-1}\right)^{\alpha} e^{x t}\left(1-x_{1} t\right)^{-\alpha_{1}}\left(1-x_{2} t\right)^{-\alpha_{2}}=\sum_{n=0}^{\infty} B g_{n}^{\left(\alpha \mid \alpha_{1}, \alpha_{2}\right)}\left(x \mid x_{1}, x_{2}\right) t^{n}
$$

so that

$$
{ }_{B} g_{n}^{\left(\alpha \mid \alpha_{1}, \alpha_{2}\right)}\left(x \mid x_{1}, x_{2}\right)=\sum_{m=0}^{n} g_{m}^{\left(\alpha_{1}, \alpha_{2}\right)}\left(x_{1}, x_{2}\right) \frac{B_{n-m}^{(\alpha)}(x)}{(n-m) !} .
$$

Setting $x=0$ in (2.4), the result reduces to

$$
\left(\frac{t}{e^{t}-1}\right)^{\alpha}\left(1-x_{1} t\right)^{-\alpha_{1}}\left(1-x_{2} t\right)^{-\alpha_{2}}=\sum_{n=0}^{\infty} B g_{n}^{\left(\alpha \mid \alpha_{1}, \alpha_{2}\right)}\left(x_{1}, x_{2}\right) t^{n}
$$

where

$$
{ }_{B} g_{n}^{\left(\alpha \mid \alpha_{1}, \alpha_{2}\right)}\left(0 \mid x_{1}, x_{2}\right)={ }_{B} g_{n}^{\left(\alpha \mid \alpha_{1}, \alpha_{2}\right)}\left(x_{1}, x_{2}\right) .
$$


Theorem 2.1. The following implicit summation formula for 2-variable Hermite polynomials $h_{n}^{\left(\alpha_{1}, \alpha_{2}\right)}\left(x_{1}, x_{2}\right)$ holds true:

$$
h_{n}^{\left(\alpha_{1}, \alpha_{2}\right)}\left(x_{1}, x_{2}\right)=\sum_{m=0}^{n} B H_{n-m}^{\left(\alpha_{1}, \alpha_{2}\right)}\left(x \mid x_{1}, x_{2}\right) \frac{(-x)^{m}}{m !} .
$$

Proof. For $\alpha=0$ in (2.3), we have

$$
e^{-x t} \sum_{n=0}^{\infty} B H_{n}^{\left(\alpha_{1}, \alpha_{2}\right)}\left(x \mid x_{1}, x_{2}\right) t^{n}=\left(1-x_{1} t\right)^{-\alpha_{1}}\left(1-x_{2} t^{2}\right)^{-\alpha_{2}},
$$

which gives

$$
\sum_{m=0}^{\infty} \frac{(-x)^{m} t^{m}}{m !} \sum_{n=0}^{\infty} B H_{n}^{\left(\alpha_{1}, \alpha_{2}\right)}\left(x \mid x_{1}, x_{2}\right) t^{n}=\sum_{n=0}^{\infty} h_{n}^{\left(\alpha_{1}, \alpha_{2}\right)}\left(x_{1}, x_{2}\right) t^{n} .
$$

Replacing $n$ by $n-m$ in the left hand side, we have

$$
\sum_{n=0}^{\infty} \sum_{m=0}^{n} B H_{n-m}^{\left(\alpha_{1}, \alpha_{2}\right)}\left(x \mid x_{1}, x_{2}\right) \frac{(-x)^{m}}{m !} t^{n}=\sum_{n=0}^{\infty} h_{n}^{\left(\alpha_{1}, \alpha_{2}\right)}\left(x_{1}, x_{2}\right) t^{n} .
$$

Comparing the coefficients of $t^{n}$, we get (2.5).

Theorem 2.2. For the Lagrange-Hermite-Bernoulli polynomials, the following implicit summation formula holds true:

$$
{ }_{B} H_{n}^{\left(\alpha \mid \alpha_{1}, \alpha_{2}\right)}\left(x+y \mid x_{1}, x_{2}\right)=\sum_{m=0}^{n} B H_{n-m}^{\left(\alpha \mid \alpha_{1}, \alpha_{2}\right)}\left(x \mid x_{1}, x_{2}\right) \frac{y^{m}}{m !} .
$$

Proof. From the definition (2.3), we have

$$
\begin{aligned}
\sum_{n=0}^{\infty}{ }_{B} H_{n}^{\left(\alpha \mid \alpha_{1}, \alpha_{2}\right)}\left(x+y \mid x_{1}, x_{2}\right) t^{n} & =\left(\frac{t}{e^{t}-1}\right)^{\alpha} e^{x t} e^{y t}\left(1-x_{1} t\right)^{-\alpha_{1}}\left(1-x_{2} t^{2}\right)^{-\alpha_{2}} \\
& =\sum_{n=0}^{\infty} B H_{n}^{\left(\alpha \mid \alpha_{1}, \alpha_{2}\right)}\left(x \mid x_{1}, x_{2}\right) t^{n} \sum_{m=0}^{\infty} y^{m} \frac{t^{m}}{m !}
\end{aligned}
$$

Replacing $n$ by $n-m$ in the above equality and comparing the coefficients of $t^{n}$, we get the equality (2.6).

Theorem 2.3. For the Lagrange-Hermite-Bernoulli polynomials, the following implicit summation formula holds true:

$$
{ }_{B} H_{n}^{\left(\alpha+\beta \mid \alpha_{1}, \alpha_{2}\right)}\left(x+y \mid x_{1}, x_{2}\right)=\sum_{m=0}^{n}{ }_{B} H_{n-m}^{\left(\alpha \mid \alpha_{1}, \alpha_{2}\right)}\left(x \mid x_{1}, x_{2}\right) B_{m}^{(\beta)}(y) \frac{1}{m !} .
$$

Proof. Using the definition (2.3), we have

$$
\sum_{n=0}^{\infty}{ }_{B} H_{n}^{\left(\alpha+\beta \mid \alpha_{1}, \alpha_{2}\right)}\left(x+y \mid x_{1}, x_{2}\right) t^{n}
$$




$$
\begin{aligned}
& =\left(\frac{t}{e^{t}-1}\right)^{\alpha+\beta} e^{(x+y) t}\left(1-x_{1} t\right)^{-\alpha_{1}}\left(1-x_{2} t^{2}\right)^{-\alpha_{2}} \\
& =\sum_{n=0}^{\infty} B H_{n}^{\left(\alpha \mid \alpha_{1}, \alpha_{2}\right)}\left(x \mid x_{1}, x_{2}\right) t^{n} \sum_{m=0}^{\infty} B_{m}^{(\beta)}(y) \frac{t^{m}}{m !} .
\end{aligned}
$$

Replacing $n$ by $n-m$ in the above equality, we have

$$
\sum_{n=0}^{\infty}{ }_{B} H_{n}^{\left(\alpha+\beta \mid \alpha_{1}, \alpha_{2}\right)}\left(x+y \mid x_{1}, x_{2}\right) t^{n}=\sum_{n=0}^{\infty} \sum_{m=0}^{n}{ }_{B} H_{n-m}^{\left(\alpha \mid \alpha_{1}, \alpha_{2}\right)}\left(x \mid x_{1}, x_{2}\right) B_{m}^{(\beta)}(y) \frac{t^{n}}{m !} .
$$

Comparing the coefficients of $t^{n}$, we get (2.7).

Theorem 2.4. For the Lagrange-Hermite-Bernoulli polynomials, the following implicit summation formula holds true:

$$
{ }_{B} H_{n}^{\left(\alpha \mid \alpha_{1}, \alpha_{2}\right)}\left(x \mid x_{1}, x_{2}\right)=\sum_{m=0}^{n}{ }_{B} H_{n-m}^{\left(\alpha \mid \alpha_{1}, \alpha_{2}\right)}\left(x-z \mid x_{1}, x_{2}\right) \frac{z^{m}}{m !} .
$$

Proof. By exploiting the generating function (2.3), we have

$$
\begin{aligned}
\sum_{n=0}^{\infty}{ }_{B} H_{n}^{\left(\alpha \mid \alpha_{1}, \alpha_{2}\right)}\left(x \mid x_{1}, x_{2}\right) t^{n} & =\left(\frac{t}{e^{t}-1}\right)^{\alpha} e^{(x-z) t} e^{z t}\left(1-x_{1} t\right)^{-\alpha_{1}}\left(1-x_{2} t^{2}\right)^{-\alpha_{2}} \\
& =\sum_{n=0}^{\infty} B H_{n}^{\left(\alpha \mid \alpha_{1}, \alpha_{2}\right)}\left(x-z \mid x_{1}, x_{2}\right) t^{n} \sum_{m=0}^{\infty} z^{m} \frac{t^{m}}{m !} .
\end{aligned}
$$

Replacing $n$ by $n-m$ in the above equality, we have

$$
\sum_{n=0}^{\infty}{ }_{B} H_{n}^{\left(\alpha \mid \alpha_{1}, \alpha_{2}\right)}\left(x \mid x_{1}, x_{2}\right) t^{n}=\sum_{n=0}^{\infty} \sum_{m=0}^{n} B H_{n-m}^{\left(\alpha \mid \alpha_{1}, \alpha_{2}\right)}\left(x-z \mid x_{1}, x_{2}\right) z^{m} \frac{t^{n}}{m !} .
$$

Comparing the coefficients of $t^{n}$, we get the result (2.8).

Theorem 2.5. For the Lagrange-Hermite-Bernoulli polynomials, the following implicit summation formula holds true:

$$
{ }_{B} H_{n}^{\left(\alpha+\beta \mid \alpha_{1}, \alpha_{2}\right)}\left(x \mid x_{1}, x_{2}\right)=\sum_{m=0}^{n}{ }_{B} H_{n-m}^{\left(\alpha \mid \alpha_{1}, \alpha_{2}\right)}\left(z \mid x_{1}, x_{2}\right) \frac{B_{m}^{(\beta)}(x-z)}{m !} .
$$

Proof. Going back to the generating function (2.3), the use of (2.2) gives

$$
\begin{aligned}
& \sum_{n=0}^{\infty} B_{B} H_{n}^{\left(\alpha+\beta \mid \alpha_{1}, \alpha_{2}\right)}\left(x \mid x_{1}, x_{2}\right) t^{n} \\
& \quad=\left(\frac{t}{e^{t}-1}\right)^{\alpha} e^{z t}\left(1-x_{1} t\right)^{-\alpha_{1}}\left(1-x_{2} t^{2}\right)^{-\alpha_{2}}\left(\frac{t}{e^{t}-1}\right)^{\beta} e^{(x-z) t} \\
& \quad=\sum_{n=0}^{\infty}{ }_{B} H_{n}^{\left(\alpha \mid \alpha_{1}, \alpha_{2}\right)}\left(z \mid x_{1}, x_{2}\right) t^{n} \sum_{m=0}^{\infty} \frac{B_{m}^{(\beta)}(x-z) t^{m}}{m !} .
\end{aligned}
$$


Replacing $n$ by $n-m$ in the above equation and comparing the coefficients of $t^{n}$, we get (2.9).

Theorem 2.6. For the Lagrange-Hermite-Bernoulli polynomials, the following implicit summation formula holds true:

$$
\sum_{m=0}^{n}{ }_{B} H_{n-m}^{\left(\alpha \mid \alpha_{1}, \alpha_{2}\right)}\left(x \mid x_{1}, x_{2}\right) h_{m}^{\left(\beta_{1}, \beta_{2}\right)}\left(x_{1}, x_{2}\right)={ }_{B} H_{n}^{\left(\alpha \mid \alpha_{1}+\beta_{1}, \alpha_{2}+\beta_{2}\right)}\left(x \mid x_{1}, x_{2}\right) .
$$

Proof. Using (2.4) and (1.4), we have

$$
\begin{aligned}
& \sum_{n=0}^{\infty}{ }_{B} H_{n}^{\left(\alpha \mid \alpha_{1}+\beta_{1}, \alpha_{2}+\beta_{2}\right)}\left(x \mid x_{1}, x_{2}\right) t^{n} \\
& \quad=\left(\frac{t}{e^{t}-1}\right)^{\alpha} e^{x t}\left(1-x_{1} t\right)^{-\alpha_{1}-\beta_{1}}\left(1-x_{2} t^{2}\right)^{-\alpha_{2}-\beta_{2}} \\
& \quad=\sum_{n=0}^{\infty}{ }_{B} H_{n}^{\left(\alpha \mid \alpha_{1}, \alpha_{2}\right)}\left(x \mid x_{1}, x_{2}\right) t^{n} \sum_{m=0}^{\infty} h_{m}^{\left(\beta_{1}, \beta_{2}\right)}\left(x_{1}, x_{2}\right) t^{m} .
\end{aligned}
$$

Replacing $n$ by $n-m$ in the above equality, we have

$$
\begin{gathered}
\sum_{n=0}^{\infty} \sum_{m=0}^{n} B H_{n-m}^{\left(\alpha \mid \alpha_{1}, \alpha_{2}\right)}\left(x \mid x_{1}, x_{2}\right) h_{m}^{\left(\beta_{1}, \beta_{2}\right)}\left(x_{1}, x_{2}\right) t^{n} \\
=\sum_{n=0}^{\infty}{ }_{B} H_{n}^{\left(\alpha \mid \alpha_{1}+\beta_{1}, \alpha_{2}+\beta_{2}\right)}\left(x \mid x_{1}, x_{2}\right) t^{n} .
\end{gathered}
$$

Comparing the coefficients of $t^{n}$, we get the equality (2.10).

Theorem 2.7. The following implicit summation formula, involving the Lagrange-Hermite-Bernoulli polynomials and Lagrange-Bernoulli polynomials, holds true:

$$
\sum_{m=0}^{n} B H_{n-m}^{\left(\alpha \mid \alpha_{1}, \alpha_{2}\right)}\left(x \mid x_{1}, x_{2}\right) \frac{(\beta)_{m} y^{m}}{m !}=\sum_{m=0}^{n} B g_{n-m}^{\left(\alpha \mid \alpha_{1}, \beta\right)}\left(x \mid x_{1}, y\right) \frac{\left(x_{2}\right)^{m}\left(\alpha_{2}\right)_{m}}{m !} .
$$

Proof. We start with the generating function (2.3). Multiplying both sides by $(1-y t)^{-\beta}$ and interpreting the result, using (2.4) and series expansion of $(1-y t)^{-\beta}$, we get the required result.

\section{Generalized Bernoulli-Miller-Lee polynomials}

The definitions (2.3) and (2.4) can be exploited in a number of ways. As a first example, we set $\alpha=\alpha_{2}=0, \alpha_{1}=m+1, x_{1}=1$ in (2.3) to get

$$
e^{x t}(1-t)^{-m-1}=\sum_{n=0}^{\infty} G_{n}^{(m)}(x) t^{n}, \quad|t|<1 .
$$


Here $G_{n}^{(m)}(x)$ are called the Miller-Lee polynomials [3, p. 21].

Another example is the definition of Bernoulli-Hermite-Miller-Lee polynomials ${ }_{B} H G_{n}^{(m, \alpha)}(x, y)$ given by the generating function

$$
\left(\frac{t}{e^{t}-1}\right)^{\alpha} \frac{e^{x t+y t^{2}}}{(1-t)^{m+1}}=\sum_{n=0}^{\infty}{ }_{B} H G_{n}^{(m, \alpha)}(x, y) \frac{t^{n}}{n !}
$$

which, for $\alpha=0$, reduces to

$$
\frac{e^{x t+y t^{2}}}{(1-t)^{m+1}}=\sum_{n=0}^{\infty}{ }_{H} G_{n}^{(m)}(x, y) \frac{t^{n}}{n !}
$$

where ${ }_{H} G_{n}^{(m)}(x, y)$ are called the Hermite-Miller-Lee polynomials.

Putting $y=0$ into (3.1) gives

$$
\left(\frac{t}{e^{t}-1}\right)^{\alpha} \frac{e^{x t}}{(1-t)^{m+1}}=\sum_{n=0}^{\infty}{ }_{B} G_{n}^{(m, \alpha)}(x) \frac{t^{n}}{n !}
$$

where ${ }_{B} G_{n}^{(m, \alpha)}(x)$ are called the Bernoulli-Miller-Lee polynomials.

Theorem 3.1. The following implicit summation formula, involving the Lagrange-Hermite-Bernoulli polynomials ${ }_{B} H_{n}^{\left(\alpha \mid \alpha_{1}, \alpha_{2}\right)}\left(x \mid x_{1}, x_{2}\right)$, BernoulliMiller-Lee polynomials ${ }_{B} G_{n}^{(m, \alpha)}(x)$, and Miller-Lee polynomials $G_{n}^{(m)}(x)$, holds true:

$$
\begin{aligned}
{ }_{B} G_{n}^{(m, \alpha)}(x) & =n ! \sum_{r=0}^{n} B_{n-r}^{(\alpha)} G_{r}^{(m)}(x) \frac{1}{(n-r) !} \\
& =n ! \sum_{r=0}^{\left[\frac{n}{2}\right]} \frac{\left(-\alpha_{2}\right)_{r}\left(x_{2}\right)^{r}}{r !}{ }_{B} H_{n-2 r}^{\left(\alpha \mid m+1, \alpha_{2}\right)}\left(x \mid 1, x_{2}\right) .
\end{aligned}
$$

Proof. Setting $x_{1}=1$ and $\alpha_{1}=m+1$ in (2.3), and using (3.2), we have

$$
\begin{aligned}
\left(\frac{t}{e^{t}-1}\right)^{\alpha} e^{x t}(1-t)^{-m-1} & =\sum_{n=0}^{\infty}{ }_{B} G_{n}^{(m, \alpha)}(x) \frac{t^{n}}{n !} \\
& =\left(1-x_{2} t^{2}\right)^{\alpha_{2}} \sum_{n=0}^{\infty}{ }_{B} H_{n}^{\left(\alpha \mid m+1, \alpha_{2}\right)}\left(x \mid 1, x_{2}\right) t^{n},
\end{aligned}
$$

which, using the binomial expansion, takes the form

$$
\sum_{n=0}^{\infty} B_{n}^{(\alpha)} \frac{t^{n}}{n !} \sum_{r=0}^{\infty} G_{r}^{(m)}(x) t^{r}=\sum_{r=0}^{\infty} \frac{\left(-\alpha_{2}\right)_{r}\left(x_{2}\right)^{r} t^{2 r}}{r !} \sum_{n=0}^{\infty}{ }_{B} H_{n}^{\left(\alpha \mid m+1, \alpha_{2}\right)}\left(x \mid 1, x_{2}\right) t^{n} .
$$

Now

$$
\sum_{n=0}^{\infty}{ }_{B} G_{n}^{(m, \alpha)}(x) \frac{t^{n}}{n !}=\sum_{n=0}^{\infty} \sum_{r=0}^{n} B_{n-r}^{(\alpha)} G_{r}^{(m)}(x) \frac{t^{n}}{(n-r) !}
$$




$$
=\sum_{n=0}^{\infty} \sum_{r=0}^{\left[\frac{n}{2}\right]} \frac{\left(-\alpha_{2}\right)_{r}\left(x_{2}\right)^{r}}{r !}{ }_{B} H_{n-2 r}^{\left(\alpha \mid m+1, \alpha_{2}\right)}\left(x \mid 1, x_{2}\right) t^{n} .
$$

Finally, comparing the coefficients of $t^{n}$, we get (3.3).

Remark 3.1. Equality (3.3) is obviously a series representation of the Bernoulli-Miller-Lee polynomials ${ }_{B} G_{n}^{(m, \alpha)}(x)$ linking Lagrange-HermiteBernoulli and Miller-Lee polynomials.

Theorem 3.2. The following implicit summation formula, involving the Lagrange-Hermite-Bernoulli polynomials ${ }_{B} H_{n}^{\left(\alpha \mid \alpha_{1}, \alpha_{2}\right)}\left(x \mid x_{1}, x_{2}\right)$ and MillerLee polynomials $G_{n}^{(m)}(x)$, holds true:

$$
{ }_{B} H_{n}^{\left(\alpha \mid \alpha_{1}+m+1, \alpha_{2}\right)}\left(x+y \mid x_{1}, x_{2}\right)=\sum_{r=0}^{n}{ }_{B} H_{n-r}^{\left(\alpha \mid \alpha_{1}, \alpha_{2}\right)}\left(y \mid x_{1}, x_{2}\right) G_{r}^{(m)}\left(\frac{x}{x_{1}}\right) x_{1}^{r} .
$$

Proof. By replacing $x$ by $x+y$ and $\alpha_{1}$ by $\alpha_{1}+m+1$ in (2.3), we have

$$
\begin{aligned}
& \left(\frac{t}{e^{t}-1}\right)^{\alpha} e^{(x+y) t}\left(1-x_{1} t\right)^{-m-1}\left(1-x_{1} t\right)^{-\alpha_{1}}\left(1-x_{2} t^{2}\right)^{-\alpha_{2}} \\
& \quad=\sum_{n=0}^{\infty} B H_{n}^{\left(\alpha \mid \alpha_{1}+m+1, \alpha_{2}\right)}\left(x+y \mid x_{1}, x_{2}\right) t^{n},
\end{aligned}
$$

which can be written as

$$
\begin{gathered}
\sum_{n=0}^{\infty}{ }_{B} H_{n}^{\left(\alpha \mid \alpha_{1}, \alpha_{2}\right)}\left(y \mid x_{1}, x_{2}\right) t^{n} \sum_{r=0}^{\infty} G_{r}^{(m)}\left(\frac{x}{x_{1}}\right) x_{1}^{r} t^{r} \\
=\sum_{n=0}^{\infty} B H_{n}^{\left(\alpha \mid \alpha_{1}+m+1, \alpha_{2}\right)}\left(x+y \mid x_{1}, x_{2}\right) t^{n} .
\end{gathered}
$$

Now replacing $n$ by $n-r$ in the left hand side of the above equality, we get

$$
\begin{gathered}
\sum_{n=0}^{\infty} \sum_{r=0}^{n} B H_{n-r}^{\left(\alpha \mid \alpha_{1}, \alpha_{2}\right)}\left(y \mid x_{1}, x_{2}\right) G_{r}^{(m)}\left(\frac{x}{x_{1}}\right) x_{1}^{r} t^{n} \\
=\sum_{n=0}^{\infty} B H_{n}^{\left(\alpha \mid \alpha_{1}+m+1, \alpha_{2}\right)}\left(x+y \mid x_{1}, x_{2}\right) t^{n} .
\end{gathered}
$$

Finally, comparing the coefficients of $t^{n}$, we get the equality (3.4).

Theorem 3.3. The following implicit summation formula, involving the Lagrange-Hermite-Bernoulli polynomials ${ }_{B} H_{n}^{\left(\alpha \mid \alpha_{1}, \alpha_{2}\right)}\left(x \mid x_{1}, x_{2}\right)$ and MillerLee polynomials $G_{n}^{(m)}(x)$, holds true:

$$
\sum_{r=0}^{n} B_{n-r H}^{(\alpha)} G_{r}^{\left(m \mid \alpha_{1}, \alpha_{2}\right)}\left(x \mid x_{1}, x_{2}\right) \frac{1}{(n-r) !}=\sum_{r=0}^{n}\left(\alpha_{1}\right)_{r} x_{1 B}^{r} H_{n-r}^{\left(\alpha \mid m+1, \alpha_{2}\right)}\left(x \mid 1, x_{2}\right) \frac{1}{r !} .
$$


Proof. For $\alpha_{1}=m+1$ and $x_{1}=1$ in (2.3), we have

$$
\left(\frac{t}{e^{t}-1}\right)^{\alpha} e^{x t}(1-t)^{-m-1}\left(1-x_{2} t^{2}\right)^{-\alpha_{2}}=\sum_{n=0}^{\infty}{ }_{B} H_{n}^{\left(\alpha \mid m+1, \alpha_{2}\right)}\left(x \mid 1, x_{2}\right) t^{n} .
$$

Multiplying both sides by $\left(1-x_{1} t\right)^{-\alpha_{1}}$, we have

$$
\begin{aligned}
& \sum_{n=0}^{\infty} B_{n}^{(\alpha)} \frac{t^{n}}{n !} \sum_{r=0}^{\infty}{ }_{H} G_{r}^{\left(m \mid \alpha_{1}, \alpha_{2}\right)}\left(x \mid x_{1}, x_{2}\right) t^{n} \\
& \quad=\left(1-x_{1} t\right)^{-\alpha_{1}} \sum_{n=0}^{\infty} B_{n} H_{n}^{\left(\alpha \mid m+1, \alpha_{2}\right)}\left(x \mid 1, x_{2}\right) t^{n} .
\end{aligned}
$$

Now, replacing $n$ by $n-r$ in the above equality, we get

$$
\begin{aligned}
\sum_{n=0}^{\infty} & \sum_{r=0}^{n} B_{n-r}^{(\alpha)} G_{r}^{\left(m \mid \alpha_{1}, \alpha_{2}\right)}\left(x \mid x_{1}, x_{2}\right) \frac{t^{n}}{(n-r) !} \\
= & \sum_{n=0}^{\infty} \sum_{r=0}^{n}\left(\alpha_{1}\right)_{r}\left(x_{1}\right)^{r}{ }_{B} H_{n-r}^{\left(\alpha \mid m+1, \alpha_{2}\right)}\left(x \mid 1, x_{2}\right) \frac{t^{n}}{r !} .
\end{aligned}
$$

Comparing the coefficients of $t^{n}$, we get the desired result.

\section{Generalized Laguerre--Bernoulli polynomials}

We shall be interested in the relation between the Lagrange-HermiteBernoulli polynomials ${ }_{B} H_{n}^{\left(\alpha \mid \alpha_{1}, \alpha_{2}\right)}\left(x \mid x_{1}, x_{2}\right)$ and Laguerre polynomials $L_{n}^{(m)}(x)$.

For $x_{2}=0, x_{1}=-1, \alpha_{1}=-m$, and $\alpha_{2}=0$ in equation (2.3), we have

$$
\left(\frac{t}{e^{t}-1}\right)^{\alpha} e^{x t}(1+t)^{m}=\sum_{n=0}^{\infty}{ }_{B} L_{n}^{(\alpha \mid m)}(x) \frac{t^{n}}{n !}
$$

where ${ }_{B} L_{n}^{(\alpha \mid m)}(x)={ }_{B} H_{n}^{(\alpha \mid-m, 0)}(x \mid-1,0)$ are called the generalized LaguerreBernoulli polynomials.

When $\alpha=0$ in (4.1), the polynomials ${ }_{B} L_{n}^{(\alpha \mid m)}(x)$ reduce to ordinary Laguerre polynomials $L_{n}^{(m)}(x)$ (see [8]).

Theorem 4.1. The following implicit summation formula, involving the Lagrange-Hermite-Bernoulli polynomials ${ }_{B} H_{n}^{\left(\alpha \mid \alpha_{1}, \alpha_{2}\right)}\left(x \mid x_{1}, x_{2}\right)$ and Laguerre polynomials $L_{n}^{(m)}(x)$, holds true:

$$
\sum_{r=0}^{n}{ }_{B} H_{n-r}^{(\alpha)}\left(x \mid x_{1}, x_{2}\right) L_{r}^{(m-r)}(y)=\sum_{r=0}^{n}(\alpha)_{r} x_{1 B}^{r} H_{n-r}^{\left(\alpha \mid-m, \alpha_{2}\right)}\left(x+y \mid-1, x_{2}\right) \frac{1}{r !} .
$$


Proof. Replacing $x$ by $x+y$ and setting $x_{1}=-1, \alpha_{1}=-m$ in (2.3), we have

$$
\left(\frac{t}{e^{t}-1}\right)^{\alpha} e^{(x+y) t}(1+t)^{m}\left(1-x_{2} t^{2}\right)^{-\alpha_{2}}=\sum_{n=0}^{\infty}{ }_{B} H_{n}^{\left(\alpha \mid-m, \alpha_{2}\right)}\left(x+y \mid-1, x_{2}\right) t^{n} .
$$

Multiplying both sides by $\left(1-x_{1} t\right)^{-\alpha_{1}}$, we have

$$
\begin{aligned}
& \left(\frac{t}{e^{t}-1}\right)^{\alpha} e^{(x+y) t}(1+t)^{m}\left(1-x_{1} t\right)^{-\alpha_{1}}\left(1-x_{2} t^{2}\right)^{-\alpha_{2}} \\
& =\left(1-x_{1} t\right)^{-\alpha_{1}} \sum_{n=0}^{\infty} B H_{n}^{\left(\alpha \mid-m, \alpha_{2}\right)}\left(x+y \mid-1, x_{2}\right) t^{n},
\end{aligned}
$$

which gives

$$
\begin{aligned}
& \sum_{n=0}^{\infty}{ }_{B} H_{n}^{(\alpha)}\left(x \mid x_{1}, x_{2}\right) t^{n} \sum_{r=0}^{\infty} L_{r}^{(m-r)}(y) t^{r} \\
& \quad=\sum_{r=0}^{\infty} \frac{(\alpha)_{r}\left(x_{1}\right)^{r} t^{r}}{r !} \sum_{n=0}^{\infty}{ }_{B} H_{n}^{\left(\alpha \mid-m, \alpha_{2}\right)}\left(x+y \mid-1, x_{2}\right) t^{n} .
\end{aligned}
$$

Replacing $n$ by $n-r$ in the above equality, we have

$$
\begin{aligned}
& \sum_{n=0}^{\infty} \sum_{r=0}^{n}{ }_{B} H_{n-r}^{(\alpha)}\left(x \mid x_{1}, x_{2}\right) L_{r}^{(m-r)}(y) t^{n} \\
& \quad=\sum_{n=0}^{\infty} \sum_{r=0}^{n}(\alpha)_{r} x_{1 B}^{r} H_{n-r}^{\left(\alpha \mid-m, \alpha_{2}\right)}\left(x+y \mid-1, x_{2}\right) \frac{t^{n}}{r !} .
\end{aligned}
$$

Comparing the coefficients of $t^{n}$, we get the desired result.

Theorem 4.2. The following implicit summation formula, involving the Lagrange-Hermite-Bernoulli polynomials ${ }_{B} H_{n}^{\left(\alpha \mid \alpha_{1}, \alpha_{2}\right)}\left(x \mid x_{1}, x_{2}\right)$ and Laguerre polynomials $L_{n}^{(m)}(x)$, holds true:

$$
\sum_{k=0}^{n} B_{n-k}^{(\alpha)}(x) L_{k}^{(m-k)}(y) \frac{1}{(n-k) !}={ }_{B} H_{n}^{(\alpha \mid-m, 0)}\left(x+y \mid-1, x_{2}\right) .
$$

Proof. Replacing $x$ by $x+y$ and setting $x_{1}=-1, \alpha_{1}=-m$, and $\alpha_{2}=0$ in (2.3), we have

$$
\left(\frac{t}{e^{t}-1}\right)^{\alpha} e^{(x+y) t}(1+t)^{m}=\sum_{n=0}^{\infty}{ }_{B} H_{n}^{(\alpha \mid-m, 0)}\left(x+y \mid-1, x_{2}\right) t^{n},
$$

and thus

$$
\sum_{n=0}^{\infty} B_{n}^{(\alpha)}(x) \frac{t^{n}}{n !} \sum_{k=0}^{\infty} L_{k}^{(m-k)}(y) t^{k}=\sum_{n=0}^{\infty}{ }_{B} H_{n}^{(\alpha \mid-m, 0)}\left(x+y \mid-1, x_{2}\right) t^{n} .
$$


Replacing $n$ by $n-k$ in the left hand side of the above equality, we have

$$
\sum_{n=0}^{\infty} \sum_{k=0}^{n} B_{n-k}^{(\alpha)}(x) L_{k}^{(m-k)}(y) \frac{t^{n}}{(n-k) !}=\sum_{n=0}^{\infty} B_{B} H_{n}^{(\alpha \mid-m, 0)}\left(x+y \mid-1, x_{2}\right) t^{n} .
$$

Comparing the coefficients of $t^{n}$, we get (4.2).

Theorem 4.3. The following implicit summation formula, involving the Lagrange-Hermite-Bernoulli polynomials ${ }_{B} H_{n}^{\left(\alpha \mid \alpha_{1}, \alpha_{2}\right)}\left(x \mid x_{1}, x_{2}\right)$ and Laguerre polynomials $L_{n}^{(m)}(x)$, holds true:

$$
\begin{aligned}
& \sum_{k=0}^{n}{ }_{B} H_{n-k}^{\left(\alpha \mid-m+\alpha_{1}, \alpha_{2}\right)}\left(x \mid x_{1}, x_{2}\right)\left(-x_{1}\right)^{k} L_{k}^{(m-k)}\left(y / x_{1}\right) \\
& \quad={ }_{B} H_{n}^{\left(\alpha \mid-m+\alpha_{1}, \alpha_{2}\right)}\left(x-y \mid x_{1}, x_{2}\right) .
\end{aligned}
$$

Proof. Replacing $\alpha_{1}$ by $\alpha_{1}-m$ and $x$ by $x-y$ in (2.3), we have

$$
\begin{aligned}
& \sum_{n=0}^{\infty}{ }_{H} B_{n}^{\left(\alpha \mid-m+\alpha_{1}, \alpha_{2}\right)}\left(x \mid x_{1}, x_{2}\right) t^{n} \sum_{k=0}^{\infty}\left(-x_{1}\right)^{k} t^{k} L_{k}^{(m-k)}\left(y / x_{1}\right) \\
& =\sum_{n=0}^{\infty} B_{B} H_{n}^{\left(\alpha \mid-m+\alpha_{1}, \alpha_{2}\right)}\left(x-y \mid x_{1}, x_{2}\right) t^{n} .
\end{aligned}
$$

Replacing $n$ by $n-k$ in the left hand side of the above equality, we have

$$
\begin{aligned}
\sum_{n=0}^{\infty} & \sum_{k=0}^{n} H B_{n-k}^{\left(\alpha \mid-m+\alpha_{1}, \alpha_{2}\right)}\left(x \mid x_{1}, x_{2}\right)\left(-x_{1}\right)^{k} L_{k}^{(m-k)}\left(y / x_{1}\right) t^{n} \\
= & \sum_{n=0}^{\infty} B H_{n}^{\left(\alpha \mid-m+\alpha_{1}, \alpha_{2}\right)}\left(x-y \mid x_{1}, x_{2}\right) t^{n} .
\end{aligned}
$$

Comparing the coefficients of $t^{n}$, we get the desired equality.

Theorem 4.4. The following implicit summation formula, involving the Lagrange-Hermite-Bernoulli polynomials ${ }_{B} H_{n}^{\left(\alpha \mid \alpha_{1}, \alpha_{2}\right)}\left(x \mid x_{1}, x_{2}\right)$ and Laguerre polynomials $L_{n}^{(m)}(x)$, holds true:

$$
\sum_{k=0}^{n} B_{n-k}^{(\alpha)}(x) L_{k}^{(m-k)}(y) \frac{1}{(n-k) !}={ }_{B} H_{n}^{(\alpha \mid-m, 0)}\left(x+y \mid-1, x_{2}\right) .
$$

Proof. Setting $x_{1}=-1, \alpha_{1}=-m, \alpha_{2}=0$, and replacing $x$ by $x-y$ in (2.3), we have

$$
\sum_{n=0}^{\infty} B_{n}^{(\alpha)}(x) \frac{t^{n}}{n !} \sum_{k=0}^{\infty} L_{k}^{(m-k)}(-y) t^{k}=\sum_{n=0}^{\infty} B_{n}^{(\alpha \mid-m, 0)}\left(x-y \mid-1, x_{2}\right) t^{n} .
$$


Replacing $n$ by $n-k$ in the left hand side of the above equality, we have

$$
\sum_{n=0}^{\infty} \sum_{k=0}^{n} B_{n-k}^{(\alpha)}(x) L_{k}^{(m-k)}(-y) \frac{t^{n}}{(n-k) !}=\sum_{n=0}^{\infty}{ }_{B} H_{n}^{(\alpha \mid-m, 0)}\left(x-y \mid-1, x_{2}\right) t^{n} .
$$

Finally, replacing $y$ by $-y$ and comparing the coefficients of $t^{n}$, we get (4.3).

Theorem 4.5. The following implicit summation formula, involving the Lagrange-Hermite-Bernoulli polynomials ${ }_{B} H_{n}^{\left(\alpha \mid \alpha_{1}, \alpha_{2}\right)}\left(x \mid x_{1}, x_{2}\right)$ and Laguerre polynomials $L_{n}^{(m)}(x)$, holds true:

$$
\sum_{r=0}^{n} B_{n-r}^{(\alpha)}(y)(-x)^{n} L_{r}^{(m-r)}(z) \frac{1}{(n-r) !}={ }_{B} H_{n}^{(\alpha \mid-m, 0)}\left(y-z \mid-1, x_{2}\right)(-x)^{n} .
$$

Proof. For $x_{1}=-1, \alpha_{1}=-m, \alpha_{2}=0, x \longrightarrow y-x$ in (2.3), we have

$$
\left(\frac{t}{e^{t}-1}\right)^{\alpha} e^{(y-x) t}(1+t)^{m}=\sum_{n=0}^{\infty} B H_{n}^{(\alpha \mid-m, 0)}\left(y-x \mid-1, x_{2}\right) t^{n} .
$$

Writing $-z t$ instead of $t$ in the above equality, and after that, replacing $x$ by $z$ and $z$ by $x$, we have

$$
\left(\frac{-x t}{e^{-x t}-1}\right)^{\alpha} e^{-x y t}(1-x t)^{m} e^{z x t}=\sum_{n=0}^{\infty}{ }_{B} H_{n}^{(\alpha \mid-m, 0)}\left(y-z \mid-1, x_{2}\right)(-x t)^{n} .
$$

This gives

$$
\begin{aligned}
& \sum_{n=0}^{\infty} B_{n}^{(\alpha)}(y) \frac{(-x t)^{n}}{n !} \sum_{r=0}^{\infty}(-x t)^{r} L_{r}^{(m-r)}(z) \\
& =\sum_{n=0}^{\infty} B_{n} H_{n}^{(\alpha \mid-m, 0)}\left(y-z \mid-1, x_{2}\right)(-x t)^{n} .
\end{aligned}
$$

Replacing $n$ by $n-r$ in the left hand side of the above equality, we have

$$
\begin{aligned}
\sum_{n=0}^{\infty} & \sum_{r=0}^{n} B_{n-r}^{(\alpha)}(y)(-x)^{n} L_{r}^{(m-r)}(z) \frac{t^{n}}{(n-r) !} \\
= & \sum_{n=0}^{\infty} B_{n} H_{n}^{(\alpha \mid-m, 0)}\left(y-z \mid-1, x_{2}\right)(-x t)^{n} .
\end{aligned}
$$

Finally, comparing the coefficients of $t^{n}$, we get the desired equality. 
Theorem 4.6. The following implicit summation formula, involving the Lagrange-Hermite-Bernoulli polynomials ${ }_{B} H_{n}^{\left(\alpha \mid \alpha_{1}, \alpha_{2}\right)}\left(x \mid x_{1}, x_{2}\right)$ and generalized Laguerre-Bernoulli polynomials ${ }_{B} L_{n}^{(m)}(x)$, holds true:

$$
\sum_{r=0}^{n}{ }_{B} L_{n-r}^{(\alpha \mid m)}(x)_{B} L_{r}^{(\beta \mid k)}(y) \frac{1}{(n-r) ! r !}={ }_{B} H_{n}^{(\alpha+\beta \mid-m-k, 0)}\left(x+y \mid-1, x_{2}\right) .
$$

Proof. Replacing $\alpha$ by $\alpha+\beta, x$ by $x+y$, and setting $x_{1}=-1, \alpha_{1}=-m-k$, $\alpha_{2}=0$ in (2.3), we have

$$
\begin{aligned}
& \left(\frac{t}{e^{t}-1}\right)^{\alpha} e^{x t}(1+t)^{m}\left(\frac{t}{e^{t}-1}\right)^{\beta} e^{y t}(1+t)^{k} \\
& \quad=\sum_{n=0}^{\infty}{ }_{B} H_{n}^{(\alpha+\beta \mid-m-k, 0)}\left(x+y \mid-1, x_{2}\right) t^{n},
\end{aligned}
$$

which leads directly to

$$
\begin{aligned}
& \sum_{n=0}^{\infty} B_{B} L_{n}^{(\alpha \mid m)}(x) \frac{t^{n}}{n !} \sum_{r=0}^{\infty} B L_{r}^{(\beta \mid k)}(y) \frac{t^{r}}{r !} \\
& \quad=\sum_{n=0}^{\infty} B H_{n}^{(\alpha+\beta \mid-m-k, 0)}\left(x+y \mid-1, x_{2}\right) t^{n} .
\end{aligned}
$$

Replacing $n$ by $n-r$ in the left hand side of the above equality, we have

$$
\begin{aligned}
& \sum_{n=0}^{\infty} \sum_{r=0}^{n}{ }_{L} B_{n-r}^{(\alpha \mid m)}(x)_{L} B_{r}^{(\beta \mid k)}(y) \frac{t^{n}}{(n-r) ! r !} \\
& \quad=\sum_{n=0}^{\infty} B_{n} H_{n}^{(\alpha+\beta \mid-m-k, 0)}\left(x+y \mid-1, x_{2}\right) t^{n} .
\end{aligned}
$$

Now, comparing the coefficients of $t^{n}$, we get (4.4).

\section{Acknowledgements}

The second author M. A. Pathan would like to thank Department of Science and Technology, Government of India, for the financial assistance for this work under project number SR/S4/MS:794/12.

\section{References}

[1] A. Altin and E. Erkus, On a multivariable extension of the Lagrange-Hermite polynomials, Integral Transforms Spec. Funct. 17 (2006), 239-244.

[2] W. Ch. C. Chan, Ch. J. Chyan, and H. M. Srivastava, The Lagrange polynomials in several variables, Integral Transforms Spec. Funct. 12(2) (2001), 139-148.

[3] G. Dattoli, S. Lorenzutta, and D. Sacchetti, Integral representations of new families of polynomials, Ital. J. Pure Appl. Math. 15 (2004), 19-28. 
[4] G. Dattoli, P. E. Ricci, and C. Cesarano, The Lagrange polynomials the associated generalizations, and the umbral calculus, Integral Transforms Spec. Funct. 14 (2003), 181-186.

[5] E. Erkus and H. M. Srivastava, A unified presentation of some families of multivariable polynomials, Integral Transforms Spec. Funct. 17 (2006), 267-273.

[6] M. A. Pathan, A new class of generalized Hermite-Bernoulli polynomials, Georgian Math. J. 19 (2012), 559-573.

[7] M. A. Pathan and W. A. Khan, Some implicit summation formulas and symmetric identities for the generalized Hermite-Bernoulli polynomials, Mediterr. J. Math. 12 (2015), 679-695.

[8] H. M. Srivastava and H. L. Manocha, A Treatise on Generating Functions, Ellis Horwood Ltd., Chichester; Halsted Press [John Wiley \& Sons. Inc.], New York, 1984.

Department of Mathematics, Faculty of Science, Integral University, LUCKNOW-226026, INDIA

E-mail address: waseem08_khan@rediffmail.com

Centre for Mathematical and Statistical Sciences (CMSS), KFri, Peechi P.O., Thrissur, Kerala-680653, India

E-mail address: mapathan@gmail.com 